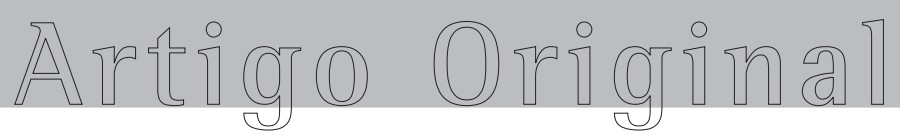

\title{
Resultado da timectomia ampliada no tratamento de pacientes com Miastenia gravis*
}

\author{
Extended Thymectomy for Treating Patients with Myasthenia Gravis (MG) \\ RAUL LOPES RUIZ JR, SAMUEL MAREK REIBSCHEID(TE SBCT), \\ ANTONIO JOSÉ MARIA CATANEO(TE SBCT), LUIS ANTONIO DE LIMA REZENDE
}

\begin{abstract}
Introdução: Diversas variações da timectomia podem ser realizadas, dentre elas a transesternal ampliada. A literatura sugere que, quanto mais extenso o procedimento para ressecção da glândula e tecidos do mediastino anterior, melhores os resultados e prognóstico.
\end{abstract}

Objetivo: Avaliar retrospectivamente a resposta à timectomia ampliada em portadores de Miastenia gravis.

Método: Foram avaliados 46 portadores de Miastenia gravis, submetidos à plasmaferese pré-operatória e à timectomia ampliada, entre agosto de 1992 e janeiro de 2003, divididos em três grupos, segundo o tempo decorrido desde o início dos sintomas: menor que 12 meses, 13 a 24 meses e maior que 25 meses.

Resultados: Trinta e um pacientes eram do sexo feminino e 15 do masculino. A média de idade foi de 30 anos. 0 tempo médio de evolução da doença foi de 26,3 meses. 0 acompanhamento ambulatorial pós-operatório foi em média de 26,6 meses. Quanto ao grau de resposta à timectomia, $89 \%$ dos pacientes tiveram boa resposta, sendo que 50\% apresentaram remissão completa. Ocorreu um óbito nesta série. 0 exame anatomopatológico demonstrou que a hiperplasia tímica foi o achado mais freqüente. Apenas 3 pacientes $(6,5 \%)$ apresentaram timomas benignos. Em 5 pacientes $(10,8 \%)$ encontramos tecido tímico extraglandular: na gordura peritímica em 2 deles, na gordura pericárdica em 1, junto ao nervo frênico esquerdo em outro e na janela aorto-pulmonar em outro.

Conclusão: A timectomia ampliada para tratamento da Miastenia gravis mostrou-se segura, eficiente, e apresentou alta porcentagem de remissão completa. Houve a detecção de tecido tímico extraglandular em alguns pacientes. Tão logo seja feito o diagnóstico, está indicada como terapêutica associada à plasmaferese pré-operatória e à medicamentosa, independentemente da idade, patologia tímica, e início dos sintomas.

J Bras Pneumol 2004; 30(2) 115-120
Background: Extended thymectomy is one of several types of thymectomy. Literature suggests that the outcome and prognosis will be enhanced by performing a more extensive resection of the gland and of the anterior mediastinum tissue.

Objective: To retrospectively evaluate response of MG patients to extended thymectomy.

Method: From August 1992 to January 2003, forty-six MG patients were submitted to preoperative plasmapheresis and extended thymectomy. According to the time elapsed since onset of symptom they were divided into three groups; $<12$ months, 13 to 24 months, and > 25 months.

Results: There were 31 female and 15 male patients, mean age 30 years and average evolution time 26.3 months. Outpatient post-operative follow-up was on the average of 26.6 months. Level of response was good, $50 \%$ had full remission and a further $39 \%$ had good response. There was one death. The most common histopathology finding was thymic hyperplasia. Only 3 patients $(6.5 \%)$ had benign thymomas; $5(10.8 \%)$ had extra glandular thymic tissue: 2 in the perithymic fat and 3 in the pericardiac fat adjacent to the left phrenic nerve and aortal-pulmonary window.

Conclusion: Treatment of MG by extended thymectomy is safe and efficient with high levels of complete remission. Extra glandular thymic tissue was found in some patients. As soon as diagnosis is completed, thymectomy is indicated together with plasmapheresis and medication, independent of age, onset of symptoms, and thymus pathology.
Descritores: Timectomia/métodos. Miastenia gravis/ patologia. Doenças neuromusculares/reabilitação.
Key words: Thymectomy/methods. Myasthenia gravis/ pathology. Neuromuscular diseases/rehabilitation. 


\section{INTRODUÇÃO}

Vários autores mostraram os benefícios da timectomia para o tratamento da Miastenia gravis $(M G)^{(1-8)}$. São realizadas diversas variações deste procedimento ${ }^{(2,3)}$, dentre elas a transesternal ampliada (ressecção total ou máxima). ${ }^{(9-14)} A$ literatura sugere que quanto mais extenso o procedimento de ressecção da glândula e tecidos do mediastino anterior, melhores os resultados e o prognóstico..$^{(1,9,15)}$ Freqüentemente são encontrados focos de tecido tímico extraglandulares, que reforçam a idéia da necessidade do procedimento ampliado. ${ }^{(1,11,15)}$ Nosso objetivo foi avaliar retrospectivamente a resposta à timectomia ampliada em portadores de MG.

\section{MÉTODO}

0 estudo foi realizado retrospectivamente para avaliar a resposta à timectomia ampliada em 46 portadores de MG, atendidos pelo Serviço de Cirurgia Torácica do Hospital das Clínicas da Faculdade de Medicina de Botucatu da Universidade Estadual Paulista (UNESP), entre agosto de 1992 e janeiro de 2003, após aprovação pelo seu Comitê de Ética em Pesquisa.

Foram avaliados os efeitos da idade, sexo, início dos sintomas, patologia tímica, severidade da doença e medicação em relação aos resultados evolutivos.

O diagnóstico da MG foi baseado na anamnese, exame físico e eletroneuromiografia. No préoperatório foram realizados os seguintes exames: radiograma simples (póstero-anterior e perfil), tomografia computadorizada de tórax, eletroneuromiografia e espirometria.

Os pacientes foram divididos em três grupos segundo o tempo decorrido desde o início dos sintomas: menor que 12 meses, de 13 a 24 meses e maior que 25 meses.

A maioria dos pacientes recebeu tratamento com anticolinesterásico (brometo de piridostigmina). Apenas um já fazia uso de corticoterapia (prednisona) no pré-operatório. Em alguns, a timectomia foi realizada sem que tenha havido o uso de quaisquer medicações específicas no pré ou pós-operatório. Em outros, a corticoterapia foi introduzida no pós-operatório, com doses que variaram entre $5 \mathrm{mg}$ e $60 \mathrm{mg}$ por dia. Esta última dosagem foi introduzida associada
Siglas e abreviaturas utilizadas neste trabalho:

Miastenia Gravis - MG

a um imunossupressor (azatioprina) em um paciente que apresentou crise miastênica no $32^{\circ}$ dia após a cirurgia.

No pré-operatório, foi utilizada a classificação clínica de Osserman ${ }^{(16)}$ (Quadro 1), dividindo-se os pacientes em cinco grupos. Foram classificados como MG forma leve os grupos 1, lla e llb; e forma grave os grupos 111 e IV.

Todos os pacientes foram submetidos a duas seções de plasmaferese, nas 72 horas que antecediam o ato operatório, sendo utilizada, para reposição volêmica, a solução de albumina humana a 5\%. Em alguns pacientes foi necessária a realização de mais uma seção de plasmaferese, no período pós-operatório, devido à piora dos sintomas.

As timectomias ampliadas foram realizadas através de esternotomia mediana, sendo que a incisão da pele se estendia por mais $3 \mathrm{~cm}$ acima da fúrcula esternal, em direção ao pescoço. A dissecção do mediastino estendia-se da porção mais inferior e anterior aos nervos frênicos até a região infra-tireoidiana e, em bloco, era removida toda a gordura pré-pericárdica, as pleuras mediastinais anteriores, bilateralmente, os coxins gordurosos dos seios cárdio-frênicos, toda a gordura próxima e anterior aos nervos frênicos e a glândula tímica. A seguir, era realizada a dissecção, delicada e cuidadosa, para a remoção de toda a gordura do recesso cavo-aórtico, da emergência dos vasos cefálicos e da região do espaço retro-tireoidiano. Este era abordado através da porção mais superior da incisão. Com o auxílio de um afastador, tracionávamos a pele e o tecido celular subcutâneo, permitindo uma boa exposição. Realizava-se a drenagem pleural, bilateralmente, através do quinto espaço intercostal, utilizando drenos de polivil multifenestrados, sob selo d'água, os quais foram retirados entre 24 e 48 horas no pós-operatório.

Todos os pacientes foram submetidos à anestesia geral inalatória com respiração controlada, monitorização da oximetria, capnografia, batimentos cardíacos e avaliações 
seriadas dos gases arteriais. Para analgesia no pósoperatório utilizamos o bloqueio com cateter peridural através da infusão contínua de agentes anestésicos por 72 horas.

A grande maioria dos pacientes foi extubada nas primeiras 24 horas do pós operatório.

No acompanhamento pós-operatório, a resposta à timectomia foi graduada segundo a classificação de Keynes (Quadro 2). ${ }^{(17)}$

\section{RESULTADOS}

Foram operados e avaliados 31 pacientes do sexo feminino e 15 do masculino. A média de idade foi de 30 anos, variando entre 10 e 64 anos. 0 tempo médio de evolução da doença foi de 26,3 meses, variando de 3 a 120 meses. 0 acompanhamento ambulatorial pós-operatório foi em média de 26,6 meses, variando de 2 a 124 meses. Quanto ao grau de resposta à timectomia, os pacientes foram avaliados segundo a classificação de Keynes: $A=23, B=9, C=9, E=$ 4 (Tabela 1). Ocorreu um óbito nesta série decorrente de embolia pulmonar, diagnosticada clinicamente, não tendo sido realizado exame necroscópico.

Nove doentes $(19,6 \%)$ não receberam nenhuma medicação no pré ou pós-operatório. No pósoperatório $7(15,2 \%)$ pacientes receberam corticoterapia associada ou não a anticolinesterásico. Em apenas um deles houve a necessidade de associação com imunossupressor.

A Tabela 2 relaciona a resposta evolutiva com o sexo, idade, tempo decorrido desde o início dos sintomas e com a classificação clínica de Osserman.

0 exame anatomopatológico demonstrou que 3 pacientes apresentaram timomas benignos, 6 timos atróficos, 5 timos normais, 3 apresentaram
QUADRO 1

Classificação de Osserman

\begin{tabular}{|c|c|}
\hline Classe & Sintomas \\
\hline 1 & somente envolvimento ocular \\
\hline $11 \mathrm{a}$ & $\begin{array}{l}\text { envolvimento muscular generalizado } \\
\text { sem repercussão respiratória }\end{array}$ \\
\hline $11 \mathrm{~b}$ & manifestações bulbares \\
\hline 111 & $\begin{array}{l}\text { rápida progressão da doença bulbar, } \\
\text { generalizada com fraqueza da } \\
\text { musculatura respiratória }\end{array}$ \\
\hline IV & $\begin{array}{l}\text { sintomas progressivos em dois anos } \\
\text { em pacientes que foram grupo } 1 \text { ou } 11\end{array}$ \\
\hline
\end{tabular}

QUADRO 2

Classificação de Keynes

\begin{tabular}{|c|c|}
\hline $\begin{array}{l}\text { Grau de } \\
\text { Resposta }\end{array}$ & Definição \\
\hline $\mathrm{A}$ & remissão completa por + de 90 dias \\
\hline B & $\begin{array}{l}\text { ausência de sintomas com diminuição } \\
\text { da dose de medicação }\end{array}$ \\
\hline C & $\begin{array}{l}\text { melhora clínica sem troca de } \\
\text { medicação }\end{array}$ \\
\hline $\mathrm{D}$ & $\begin{array}{l}\text { sem melhora clínica com a mesma } \\
\text { dose de medicação }\end{array}$ \\
\hline E & piora clínica \\
\hline
\end{tabular}

TABELA 1

Dados demográficos, início dos sintomas, classificação de Osserman e terapêutica

\begin{tabular}{lc}
\hline Pacientes (N) & 46 \\
Sexo & \\
$\quad$ Feminino & 31 \\
$\quad$ Masculino & 15 \\
ldade (anos) & \\
$\quad$ Média & 30 \\
$\quad$ Variação & $10-64$ \\
Evolução (meses) & \\
$\quad$ Média & 26,3 \\
$\quad$ Variação & $3-120$ \\
Acompanhamento (meses) & \\
$\quad$ Média & 26,6 \\
Variação & $2-124$ \\
Início dos sintomas (meses) & \\
$\quad<12$ & 24 \\
13-23 & 3 \\
$>24$ & 19 \\
Osserman & \\
l & 4 \\
lla & 20 \\
Ilb & 14 \\
III & 8 \\
IV & 0 \\
Terapêutica & \\
Medicamentosa + timectomia & 37 \\
Timectomia & 9 \\
\hline
\end{tabular}


timos fibróticos e em 29 havia hiperplasia tímica (Tabela 2). Em 5 pacientes (10,8\%) encontrou-se tecido tímico extraglandular, sendo que em 2 (4,3\%) deles na gordura peritímica e em $3(6,5 \%)$ em outras localizações: em 1, na gordura pericárdica, em outro junto ao nervo frênico esquerdo e no outro na janela aorto-pulmonar. 0 material enviado ao patologista, para análise em separado, era constituído pelo timo com sua cápsula intacta e os tecidos peritímicos, além de toda a gordura do compartimento anterior do mediastino.

Não ocorreram complicações infecciosas ou hemorrágicas da parede torácica ou da cavidade pleural. A dor não foi queixa freqüente nas primeiras 72 horas, e após este período foi fácil o seu controle com analgésicos comuns.

Apenas um paciente apresentou insuficiência respiratória, no pós-operatório imediato, e necessitou de re-intubação, permanecendo por mais três dias. Nesta série não ocorreram distúrbios de coagulação no intra ou no pós-operatório.

Em 5 (10,8\%) pacientes foi necessária mais uma seção de plasmaferese no pós-operatório devido à piora dos sintomas. Dentre estes ocorreu 1 óbito,

TABELA 2

Resposta à timectomia ampliada quanto ao sexo, faixa etária, início dos sintomas, classificação de Osserman e anatomopatológico de tecido tímico

\begin{tabular}{|c|c|c|c|c|c|c|}
\hline Resposta & A & B & C & $\mathrm{D}$ & $E$ & Total \\
\hline \multicolumn{7}{|l|}{ Sexo } \\
\hline Feminino & 16 & 7 & 6 & 0 & 1 & 30 \\
\hline Masculino & 7 & 2 & 3 & 0 & 3 & 15 \\
\hline Total & 23 & 9 & 9 & 0 & 4 & 45 \\
\hline \multicolumn{7}{|l|}{ Faixa Etária } \\
\hline$<15$ & 7 & 0 & 1 & 0 & 0 & 8 \\
\hline $15-30$ & 10 & 3 & 1 & 0 & 1 & 15 \\
\hline $30-45$ & 4 & 4 & 6 & 0 & 2 & 16 \\
\hline$>45$ & 2 & 2 & 1 & 0 & 1 & 6 \\
\hline Total & 23 & 9 & 9 & 0 & 4 & 45 \\
\hline \multicolumn{7}{|l|}{ Início Sintomas } \\
\hline$<12$ & 9 & 2 & 4 & 0 & 4 & 19 \\
\hline $13-23$ & 6 & 1 & 1 & 0 & 0 & 8 \\
\hline$>24$ & 8 & 6 & 4 & 0 & 0 & 18 \\
\hline Total & 23 & 9 & 9 & 0 & 4 & 45 \\
\hline \multicolumn{7}{|l|}{ Osserman } \\
\hline 1 & 2 & 1 & 0 & 0 & 1 & 4 \\
\hline $11 a$ & 11 & 5 & 3 & 0 & 1 & 20 \\
\hline $11 b$ & 8 & 1 & 4 & 0 & 1 & 14 \\
\hline 111 & 2 & 2 & 2 & 0 & 1 & 7 \\
\hline IV & 0 & 0 & 0 & 0 & 0 & 0 \\
\hline Total & 23 & 9 & 9 & 0 & 4 & 45 \\
\hline \multicolumn{7}{|l|}{ Anatomopatológico } \\
\hline Hiperplasia & 16 & 5 & 6 & 0 & 1 & 28 \\
\hline Extraglandular & 2 & 0 & 1 & 0 & 2 & 5 \\
\hline Timoma & 1 & 0 & 1 & 0 & 1 & 3 \\
\hline Atrófico & 1 & 0 & 2 & 0 & 2 & 5 \\
\hline Normal & 3 & 2 & 0 & 0 & 0 & 5 \\
\hline Fibrótico & 0 & 1 & 2 & 0 & 0 & 3 \\
\hline Total & 23 & 8 & 12 & 0 & 6 & 49 \\
\hline
\end{tabular}


em 2 houve remissão completa e nos demais piora clínica mesmo após a cirurgia.

\section{DISCUSSÃO}

Desde 1939 as publicações relatam a timectomia como tratamento para Miastenia gravis $^{(2)}$. Em 1988 as publicações de Jaretzki ${ }^{(9,10)}$ enfatizaram a indicação de timectomia ampliada, com a ressecção de toda a gordura do compartimento mediastinal anterior, sempre em busca de tecido tímico extraglandular, que seria a causa da manutenção dos sintomas em pacientes operados $^{(22)}$.

Nesta série a timectomia ampliada foi realizada segundo a técnica descrita por Jaretzki ${ }^{(9)}$, porém modificada. A cervicotomia transversa foi substituída pela da incisão da pele e do subcutâneo mais estendida (cervicotomia longitudinal), em direção à região do espaço infratireoidiano e polos superiores da glândula tireóide $^{(23)}$, o que permitiu uma boa abordagem para a dissecção dos tecidos que poderiam conter resquícios embrionários do timo.

Não ocorreram complicações infecciosas da parede torácica ou das cavidades pleurais, talvez pela baixa freqüência de pacientes em corticoterapia no pré-operatório. Estudo da literatura mostrou uma leve tendência, não significativa estatisticamente, de aumento da incidência de complicações infecciosas em pacientes inunossuprimidos (14\%) quando comparados com pacientes imunocompetentes (9\%) ${ }^{(23)}$.

Observamos boa resposta à timectomia em 41 pacientes (89\%) (Tabela 2), resultado concordante com a literatura, que relata de $80 \%$ a $94 \%$ de boa resposta pós-operatória ${ }^{(7,24-27)}$. A remissão completa sem uso de qualquer medicação específica foi observada em 23 pacientes (50\%). Esta porcentagem pode ser considerada relativamente elevada quando comparada a outros estudos, que apresentaram remissões completas entre 12,7\% e $61 \% 0^{(1,7,8,24-27,30)}$. Acreditamos que nossos bons resultados estão relacionados com a maior extração de tecido tímico, possível através de um procedimento ampliado, além da realização de plasmaferese pré-operatória, a qual reduz a concentração de anticorpos contra receptores nicotínicos da acetilcolina na junção neuromuscular. 1sto propicia melhores condições respiratórias no pré e no pós-operatório, o que permite a extubação precoce destes doentes, fato comprovado neste estudo, pelo reduzido número de pacientes que apresentaram insuficiência respiratória no pós-operatório imediato ${ }^{(1)}$.

A presença de tecido tímico extraglandular ocorreu em 5 pacientes (10,8\%), e 2 destes $(4,3 \%)$ foram classificados como sem resposta (Tabela 2 ). Este fato leva-nos a confiar na idéia de Jarestski ${ }^{(11)}$ de que ressecções "máximas" levam a melhores resultados com maior freqüência de remissões completas, e os maus resultados devem-se a restos tímicos ${ }^{(1,8,22)}$ que manteriam a produção de autoanticorpos contra receptores de acetilcolina.

0 timoma benigno esteve presente em 3 pacientes $(6,5 \%)$, resultado inferior ao encontrado na literatura (entre 9\% e 28\%) ${ }^{(6,23)}$. Destes pacientes, 2 apresentaram boa resposta: 1 com remissão completa e outro com melhora clínica com a mesma dosagem de medicação do pré-operatório. 0 terceiro paciente apresentou piora clínica da MG. Nossos resultados diferem da literatura no que diz respeito às piores respostas, freqüentes nos portadores de timomas que apresentam sintomas mais precocemente e respostas piores no pós-operatório (15). Talvez esta diferença se deva aos poucos casos que apresentamos neste momento.

Existem controvérsias quanto à indicação da timectomia ou do tratamento clínico exclusivo ${ }^{(12)}$. Muitos autores acreditam que ela deve ser indicada quando houver falha no tratamento medicamentoso, enquanto que outros indicam a timectomia precoce como terapêutica primária ${ }^{(30)}$ para a MG.

Em nossa série, 9 pacientes (19,6\%) foram submetidos à timectomia sem prévio tratamento medicamentoso. Destes, 7 foram classificados como Osserman ${ }^{(16)}$ lla, 1 como llb e outro como 11l. Nos 9 pacientes ocorreu remissão completa da doença. A timectomia ampliada como terapêutica primária pareceu-nos ser capaz de propiciar maiores benefícios que o tratamento clínico exclusivo. Além disso, os riscos decorrentes do uso continuado de drogas (corticosteróides e imunossupressores) e seus efeitos colaterais indesejáveis foram minimizados.

0 período decorrido entre o início dos sintomas e a timectomia é um importante fator prognóstico ${ }^{(7,15,24,31)}$. Em nossa casuística, 4 pacientes, classificados como Osserman 1, 1la, 11b e 111, apesar de apresentarem o início dos sintomas em menos de doze meses, evoluíram com piora 
clínica após a cirurgia (Tabela 2). Talvez este resultado conflitante se deva à pequena casuística que apresentamos no momento.

Concluímos que a timectomia ampliada para o tratamento da MG é segura, eficiente, apresenta alta porcentagem de remissão completa sem o uso de medicação específica, além de poder proporcionar, como nesta série, a detecção de tecido tímico extraglandular em alguns pacientes. Acreditamos que deve ser indicada tão logo seja feito o diagnóstico de MG, como terapêutica associada à plasmaferese pré-operatória e à terapêutica medicamentosa, independentemente da idade, da patologia tímica e do tempo decorrido desde o início dos sintomas.

\section{REFERÊNCIAS}

1. Ashour M. Prevalence of ectopic thymic tissue in myasthenia gravis and its clinical significance. J Thorac Cardiovasc Surg 1995;109:632-5.

2. Blalock A, Mason MF, Morgan FJ, Riven SS. Myasthenia gravis and tumours of the thymic region. Report of a case in which the tumour was removed. Ann Surg 1939;110:544-61.

3. Blalock A. Thymectomy in the treatment of myasthenia gravis. J Thorac Surg 1944;13:316-39.

4. Bramis J. Thymectomy and myasthenia gravis. Eur J Surg 1997;163:897-902.

5. Busch C, Machens A, Pichlmeier U. Long-term outcome and quality of life after thymectomy for myasthenia gravis. Ann Surg 1996;224:225-32.

6. Goldman AJ, Herman CJR, Klesey JC. Myasthenia gravis and invasive thymoma: a 20 year experience. Neurology 1975;1021-5.

7. Hassantash SA, Ashbaugh DG, Verrier ED, Maier RV. Surgical treatment of myasthenia gravis in two major Midle East teaching hospitals: factors influencing outcome. Thorax 1996;51:193-6.

8. Huang M, King K, Hsu W, Huang B, Hsu H, Wang L, et al. Outcome of thymectomy in nonthymomatous myasthenia gravis. Surg Ginecol Obstet 1988;166:436-40.

9. Jaretzki A 111, Wolff M. "Maximal" thymectomy for myasthenia gravis. Surgical anatomy and operative technique. J Thorac Cardiovasc Surg 1988;96:711-6.

10. Jaretzki A, Penn AS, Youger DS. Maximal thymectomy for myasthenia gravis. J Thorac Cardiovasc Surg 1988;95:747-57.

11. Jaretzki A 111. Thymectomy for myasthenia gravis: an analysis of the controversies regarding technique and results. Neurology 1997;48:S52-S63.
12. Jaretzki A 11l. Outcome after transcervical thymectomy. Ann Thorac Surg 1999;67:592-601.

13. Jaretzki A 111. Transcervical-transsternal maximal thymectomy for miastenia gravis. In: Shields TW, editor. General thoracic surgery. 5th ed. Philadelphia: Lippincott Williams \& Wilkins; 2000. p.2223-31.

14. Mack MJ, Landreneau RD, Yim AP, Hazelrigg SR, Scruggs GR. Results of video-assisted thymectomy in patients with myasthenia gravis. J Thorac Cardiovasc Surg 1996;112:1352-60.

15. Masaoka A, Yamakawa Y, Niwa H, Fukai 1, Kondo S, Kobayashi $\mathrm{M}$, et al. Extended thymectomy for myasthenia gravis patients: a 20-year review. Ann Thorac Surg 1996;62:853-9.

16. Osserman KE, Genkins G. Studies in myasthenia gravis: review of twenty year experience in over 1200 patients. Mt Sinai J Med 1971;38:497-508.

17. Keynes G. Surgery of thymus gland: second thoughts. Lancet 1954;1:1197-1202.

18. Otto TJ, Strugalska H. Surgical treatment for myasthenia gravis. Thorax 1987;42:199-204.

19. Saad Jr R, Arranz CC, Dorgan N, V, Giannini JA, Botter M. Resultado da timectomia em doentes com miastenia gravis. J Pneumol 1997;23:189-92.

20. Seybold ME. Thymectomy in childhood myasthenia gravis. Ann N Y Acad Sci 1998;13:731-41.

21. Slater G, Papatestas AE, Genkins G. Thymomas in patients with myasthenia gravis. Ann Surg 1978;188:171-4.

22. Masaoka A, Monden Y, Seike Y, Tanioka T, Kagotani K. Reoperation after transcervical thymectomy for myasthenia gravis. Neurology 1982;32:83-5.

23. Bulkley GB, Bass KN, Stephenson GR, Diener-West M, George S, Reilly PA, et al. Extended cervicomediastinal thymectomy in the integratede management of myasthenia gravis. Ann Surg 1997;226:324-35.

24. Nussbaum MS, Rosenthal GJ, Samaha FJ, Grinvalsky HT, Quinlan JG, Schmerler M, et al. Managment of myasthenia gravis by extended thymectomy with anterior mediastinal dissection. Surgery 1992;112:681-8.

25. Tsuchida M, Yamato Y, Souma T, Yoshiya K, Watanabe T, Aoki T, et al. Efficacy and safety of extended thymectomy for elderly pacients with myasthenia gravis. Ann Thorac Surg 1999;67:1563-7.

26. Pego-Fernandes PM, Campos JRM, Jatene FB, Marchhiori $P$, Suso FV, Oliveira SA. Thymectomy by partial sternotomy for the treatment of myasthenia gravis. Ann Thorac Surg 2002;74:204-8.

27. Olanow CW, Wechsler AS, Sirotkin-Rosses M, Stajich J, Roses AD. Thymectomy as primary therapy in myasthenia gravis. Ann N Y Acad Sci 1987;505:595-606.

28. Nieto IP, Robledo JPP, Pajuelo MC, Montes JAR, Giron JG, Alonso JG, et al. Prognostic factors for myasthenia gravis treated by thymectomy: review of 61 cases. Ann Thorac Surg 1999;67:1568-71. 